\title{
High-gradient microelectromechanical system quadrupole electromagnets for particle beam focusing and steering
}

\author{
Jere Harrison, ' Yongha Hwang, Omeed Paydar, and Jimmy Wu \\ Department of Electrical Engineering, University of California, Los Angeles, California 90095, USA \\ Evan Threlkeld, James Rosenzweig, and Pietro Musumeci ${ }^{\dagger}$ \\ Department of Physics, University of California, Los Angeles, California 90095, USA \\ Rob Candler \\ Department of Electrical Engineering, University of California, Los Angeles, California 90095, USA \\ and California NanoSystems Institute, Los Angeles, California 90095, USA
}

(Received 14 August 2014; published 17 February 2015)

\begin{abstract}
Recent advancements in microelectromechanical system (MEMS) fabrication techniques have enabled the batch-fabrication of quadrupole MEMS electromagnets producing $100 \mathrm{mT}$-scale field across sub-mm gaps with the potential for transformational advances in the field of compact high performance charged particle focusing and steering optics. The footprint of these in-vacuum focusing and steering optics can be as small as $3 \mathrm{~mm} \times 3 \mathrm{~mm} \times 0.5 \mathrm{~mm}$. The low electromagnet impedance $(58 \mathrm{~m} \Omega, 32 \mathrm{nH}$ per pole) facilitates power-efficient operation and continuous or low duty cycle operation, and the individually controlled electromagnets allow combined dipole-quadrupole fields. Here we report on an experiment where these miniature devices have been used to focus and steer a $34 \mathrm{keV}$ electron beam from a DC photogun, demonstrating the first application of magnetic MEMS to particle beam focusing.
\end{abstract}

DOI: 10.1103/PhysRevSTAB.18.023501

PACS numbers: 41.85.Lc, 85.85.+j

\section{INTRODUCTION}

Charged particle beam optical elements such as dipoles, quadrupoles and higher order multipoles play a fundamental role in all applications of high quality electron beams throughout science and medicine, from microscopy [1] and diffraction [2] to cancer radiotherapy [3] and the production of intense and coherent X-Rays [4].

For all these demanding applications, it is critical to improve the performance and reduce the size of beam transport systems. For example, matching the particle beam width to the optimal size in free electron laser [5] and inverse Compton scattering light sources (ICS) [6-8] dramatically improves power efficiency and source brightness, but ultra-high gradient focusing and short effective magnetic length are required in order to achieve sub- $\mu \mathrm{m}$ spot sizes. In electron microscopes, stronger magnetic lenses can be used to reduce the beam size at the sample and/or increase the magnification of the system while higher order magnetic elements correct the effect of

\footnotetext{
* Corresponding author.

jere.harrison@ucla.edu

musumeci@physics.ucla.edu

rcandler@ee.ucla.edu

Published by the American Physical Society under the terms of the Creative Commons Attribution 3.0 License. Further distribution of this work must maintain attribution to the author $(s)$ and the published article's title, journal citation, and DOI.
}

aberrations on the instrument spatial resolution $[9,10]$. In plasma wakefield accelerator applications, matching the beam to the extreme strong focusing of a plasma channel necessitates very small spots at injection $[11,12]$ using very strong, very short focal length quadrupoles, and the large angular divergence leaving the laser plasma wakefield accelerator necessitates high-gradient focusing over a short distance to minimize bunch elongation and retain high peak current [13]. In multistage laser-dielectric accelerator and undulator structures, strong magnetic optics matched in size-scale to the sub-mm accelerator or undulator gap are required to realize a full scale demonstration of a light source system [14-18].

Simple electromagnet performance scaling indicates that decreasing the gap between the poles is a direct way to improve dipole field strength and quadrupole field gradient. For example, magnetic circuit analysis of a dipole electromagnet with a high-permeability yoke and no field fringing yields $B=\mu_{0} n I / 4 r$ for the field intensity, where $\mu_{0}$ is the permeability of free space, $n$ is the number of electromagnet turns on each pole, $I$ is the current driving each set of windings, and $r$ is the distance between the center of the gap and pole tip. For charged particle focusing, quadrupoles can be characterized by their effective magnetic length $L_{m}$ and normalized strength $k^{2}=q g / p$ where $q$ is the particle charge, $g$ is the field gradient, and $p$ is the beam momentum. The focal length for a single quadrupole can be written as $f^{-1}=k^{2} L_{m}$ in the thin lens 


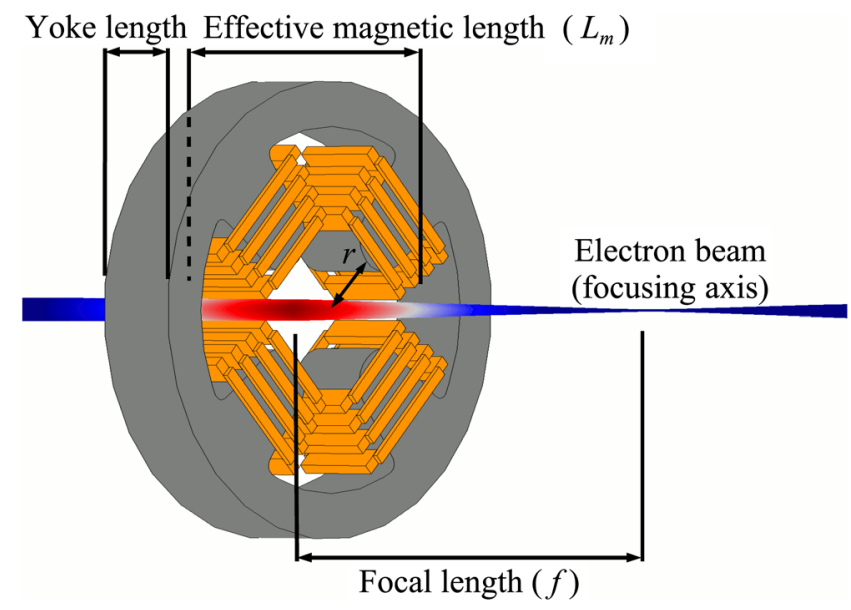

FIG. 1. Particle-tracking illustration of a $0.3 \mathrm{~mm}$ electromagnet gap radius, $0.2 \mathrm{~mm}$ yoke length MEMS quadrupole acting on an electron beam. The magnitude of the force on the electron beam is illustrated in color (e.g., red $=\max$ force). The illustration perspective shows electron beam focusing on-axis of the quadrupole; a perspective from the top would show defocusing of the electron beam.

approximation (e.g., when the focal length is greater than the effective magnetic length, $f \gg L_{m}$ ). The field gradient in a quadrupole with a high-permeability yoke neglecting fringing can be approximated as $g=2 \mu_{0} n I / r^{2}$. These parameters are illustrated in Fig. 1.

Looking at these examples, we note how the strength of the electromagnet is related inversely to the distance between the pole tips for both dipole and quadrupole fields while material parameters such as the yoke's magnetic saturation or the maximum electromagnet winding current density are not affected by scaling. Additionally, a smaller electromagnet gap reduces the total magnetic flux necessary to produce a field. This is reflected in the electromagnet design as a reduced number of winding turns, reducing the resistance, inductance, and the circuit time constant. For pulsed beams, a fast magnet response allows short-duty-cycle electromagnet switching, reducing power consumption and enabling pulse-to-pulse reconfiguration of the beam optics. As a result, scaling from traditional cm-gap out-of-vacuum magnetic optics to sub-mm-gap in-vacuum MEMS optics provides a path for ultra-highgradient focusing and fast reconfigurable beam control on a size-scale compatible with compact accelerators while reducing the size, weight, and power consumption of beam transport systems.

Reducing the electromagnet gap to MEMS-scale size places constraints on the beam source and transport system. In order to pass through the $0.1 \mathrm{~mm}$-scale good-field region of the MEMS quadrupole electromagnet, the initial beam must be smaller than the electromagnet gap or focusable below $0.1 \mathrm{~mm}$ spot-size by an upstream lens. Recently, much progress has been made in high brightness electron sources, which are now capable of producing high current electron beams with sub-mm-mrad emittances, thus enabling the development of small-aperture magnetic elements [19-21].

Due to the short effective magnetic length (sub-mm), these magnets are presently most appropriate for low energy beamlines (up to few $\mathrm{MeV}$ ). In order to extend application of MEMS magnets to high energy beams (over $100 \mathrm{MeV}$ ), new microfabrication processes must be developed to increase the effective magnetic length of the electromagnet to the $\mathrm{mm}$-scale.

In this paper, we present experimental results for the first quadrupole particle beam focusing optics fabricated using magnetic MEMS manufacturing processes. The electromagnet was manufactured and packaged at UCLA using a recently developed microfabrication process (manufacturing process described in detail in [22]) and tested in the UCLA Particle Beam Physics Laboratory. For this kind of quadrupole, linear field response to applied current is limited by saturation magnetization and maximum field strength is limited by thermal constraints; $1.1 \mathrm{~T}$ saturation magnetization for $\mathrm{Ni}_{80} \mathrm{Fe}_{20}$ and $300^{\circ} \mathrm{C}$ to reach mechanical stress limits in this work. Additionally, the achievable field gradient is set by design parameters such as the electromagnet gap distance, pole cross-section taper, yoke thickness, and yoke width. Electron beam-probe measurements for a $0.3 \mathrm{~mm}$-radius $0.055 \mathrm{~mm}$-long quadrupole electromagnet demonstrated a gradient between $47 \mathrm{~T} / \mathrm{m}$ and $58 \mathrm{~T} / \mathrm{m}$ at $1 \mathrm{~A}$ with $0.477 \mathrm{~mm}$ effective magnetic length, within $13 \%$ of the $54 \mathrm{~T} / \mathrm{m}$ predicted by simulations in the $0.143 \mathrm{~mm}$ wide good field region. Steering and focusing experiments show quadrupole saturation at $4.7 \mathrm{~A}$ and packaging failure at $5.5 \mathrm{~A}$, which prevented field characterization at the maximum currents. Optimized quadrupoles using flip-chip packaging rather than wire bonding are projected to produce multi-kT/m field gradients without packaging failure. The rapid tunability, high field gradient, and low hysteresis indicate that batch-fabricated microquadrupoles could fill a present need in particle accelerators and light sources.

\section{STANDARD APPROACHES TO QUADRUPOLE MAGNETS USED IN PARTICLE BEAM OPTICS}

Several approaches have been used by other groups to produce small-gap quadrupole optics. Permanent magnet quadrupoles (PMQ) can produce very high field gradients in a small gap without power consumption but must be hand-assembled and lack tunability. Several groups have taken this approach recently; Lim et al. demonstrated a $2.5 \mathrm{~mm}$-radius PMQ that produced $560 \mathrm{~T} / \mathrm{m}$ and Eichner et al. demonstrated a $3 \mathrm{~mm}$-radius PMQ that produced $503 \mathrm{~T} / \mathrm{m}$ for ICS and undulator experiments $[8,23]$. Another approach relies on pulsed electromagnets to produce high gradients at a low duty cycle. Winkler et al. demonstrated a $10 \mathrm{~mm}$-radius pulsed quadrupole that produced $1400 \mathrm{~T} / \mathrm{m}$ gradient at $1 \mathrm{~Hz}$ using $20 \mathrm{kA}$-pulses 


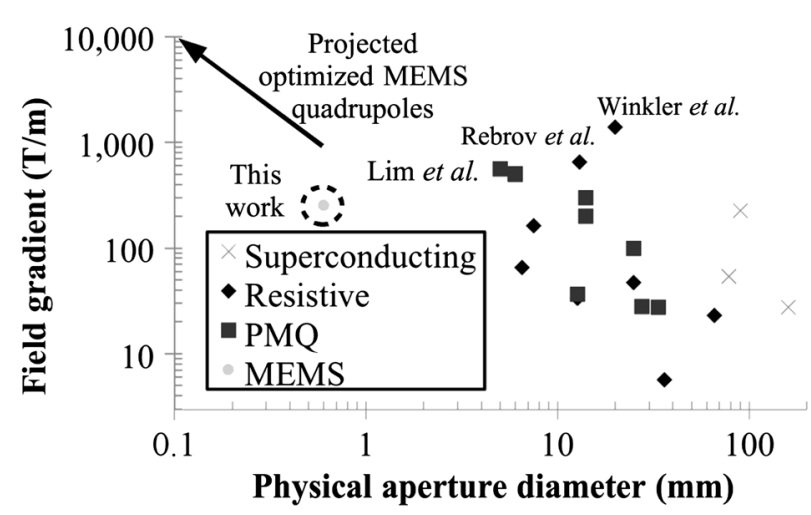

FIG. 2. Peak field gradient of superconducting and resistive electromagnet, permanent magnet (PMQ), and the MEMS quadrupoles described here. The tested MEMS quadrupole is circled and the simulated results for optimized quadrupole designs are indicated by the arrow.

[24], but the complicated power supply for this scheme has limited its utility. Another approach, taken by Rebrov et al., uses electrical discharge machining to manufacture traditional quadrupole electromagnets with a cm-scale gap and $0.01 \mathrm{~mm}$-scale geometry tolerance [25]. This produced a tunable quadrupole with $650 \mathrm{~T} / \mathrm{m}$ gradient across a $6.5 \mathrm{~mm}$-radius for ion beam analysis systems. Scaling Rebrov's approach further requires smaller and thinner beam pipes, complicating the beam system. The closestscale focusing optics to date is an in-vacuum electrostatic Einzel lens demonstrated by Shui et al. using $300 \mathrm{~V}$ across several $1 \mathrm{~mm}$-gap electrodes [26]. This approach is useful for beam energies $\mathrm{keV}$-scale and lower. Figure 2 shows the prior art in the context of magnetic gap vs peak field gradient.

\section{NOVELTY OF THIS APPROACH}

Microscale quadrupoles are manufactured by combining the fabrication techniques developed for nano- and microscale integrated circuits with special purpose MEMS processes and materials. The geometry of this quadrupole electromagnet (and thousands of devices manufactured in parallel on each substrate) is defined by a series of 2-D patterns optically defined in photosensitive polymers. A device is created as layer-by-layer extrusions of these patterns, either adding a film of material and etching it through a patterned polymer mask or adding material through a patterned polymer mold. Examples of MEMS applied to other physical systems include mm-scale accelerometers [27], image modulators [28], and atomic clocks [29].

The details of the manufacturing process for the quadrupole used in Sec. VIII are fully described in Ref. [22]. Briefly, manufacturing consists of a 3-step winding fabrication process where copper is electrochemically grown through polymer molds resulting in 3 -D coils surrounding a soft magnetic yoke. During winding fabrication, a yoke fabrication step is inserted where Permalloy 80/20 is electrochemically grown inside the incomplete windings using the process described in Ref. [30]. Between steps, layers are planarized by chemical mechanical polishing, molds are stripped by plasma etching, and the yoke and windings are separated using layers of silicon nitride grown by plasma-enhanced chemical vapor deposition. Individual quadrupoles are singulated from the substrate using a dicing saw or plasma etch.

The prototype quadrupole was required to fit within a $3 \mathrm{~mm} \times 3 \mathrm{~mm}$ footprint and layer thicknesses were required to be less than $0.06 \mathrm{~mm}$ to comply with the design rules for the fabrication run. These temporary limitations restricted the back of the electromagnet yoke to $0.2 \mathrm{~mm}$ wide and $0.055 \mathrm{~mm}$ thick. The large difference between transverse and longitudinal dimensions is common in MEMS devices and complicates the use of simple analytical approximations [31], making numerical methods preferential when computing resources are available.

\section{ANALYTICAL QUADRUPOLE APPROXIMATIONS}

Simple 2-D analysis of a quadrupole with a highpermeability yoke and a $0.3 \mathrm{~mm}$ electromagnet radius yields $112 \mathrm{~T} / \mathrm{m}$ quadrupole field gradient at $1 \mathrm{~A}$ winding current. An infinitely long yoke with hyperbolic shaped poles would produce $34 \mathrm{mT}$ field intensity at the pole tip. The effective magnetic length of a quadrupole with a finitelength yoke is often approximated by adding the radius of the quadrupole to the yoke length. The analytically approximated effective magnetic length for the prototype MEMS quadrupole with 0.055-mm-yoke length would be $0.355 \mathrm{~mm}$.

In practice, several factors lead to actual field distributions deviating from this ideal case. Yoke permeability is finite, and high-permeability materials such as our Permalloy $80 / 20$ are limited by shape anisotropy to an effective permeability far below the bulk material properties of a sphere or disk. Quadrupoles manufactured using MEMS processes are particularly sensitive to design tradeoffs that limit the pole tip shape. For example, pole tips are truncated to be manufacturable, invalidating the approximation of the magnetic equipotential lines as perfect hyperboloids. The thin yoke in MEMS electromagnets also result in significant magnetic field fringing from the pole before the tip, making it advantageous to use the entire distance between yoke and pole tip for windings to achieve the highest possible field gradients. This larger portion of the field fringing from pole surfaces relative to a quadrupole with yoke length several times as long as the electromagnet radius increases the 3-D effective magnetic length compared to analytic approximations.

A result of finite permeability in the yoke, short yoke length, and imperfect pole tip shape is that Ampère's 
Circuital Law cannot accurately approximate the flux loop as $n I=\int_{0}^{r} g r d r$. Design considerations, such as tapering the magnetic pole tips to increase the flux density at the pole tip, further complicate analytical calculation. Magnetostatic simulations using the finite element method (FEM) are commonly used to bridge the gap between analytical approximations for simplified electromagnet geometries and the complicated 3-D geometries of magnetic MEMS devices.

\section{MAGNETOSTATIC SIMULATIONS}

Static 3-D FEM simulations of the magnetic field were performed using COMSOL Multiphysics. An initial simulation of yoke and winding geometries that were designed to fit within our manufacturing limits was run with $1 \mathrm{~A}$ current in each set of windings. Mesh density in the electromagnet gap was greater than $10^{7}$ elements $/ \mathrm{mm}^{3}$ for each simulation. The peak field in the yoke or pole was used to determine the saturation current of the electromagnet and a second simulation using a nonlinear material model based on measurements of our electroplated permalloy was run for a more accurate field distribution. Table I details the results, with the prototype quadrupole electromagnet typeset bold alongside several designs with pole taper angle, yoke length, and yoke width optimized for peak gradient. The geometric details of the presented quadrupoles are the Magnet radius, the distance between the electromagnet center and pole tips, Yoke length, the physical length of the quadrupole in the longitudinal direction, and Pole taper, the angle between the pole edge and the pole center-line. The listed performance details of the quadrupoles are the Peak field gradient, the transverse magnetic field gradient along the longitudinal axis of the electromagnet when the yoke or poles are driven to saturation, Magnetic length, the normalized interaction distance of the particle beam in the field gradient, $L_{m}=\int g d z / g_{\text {peak }}$, and Winding current, the drive current in the windings that saturate the electromagnet.

Peak field gradient was calculated using the quadrupole field component of the simulated field at the edge of the $0.1 \%$ good field region, $0.072 \mathrm{~mm}$ from the center, where the sum of multi-pole field components higher than the quadrupole moment are less than $0.1 \%$ the magnitude of the quadrupole field moment. Figures 3 and 4 show a simulation of the transverse magnetic flux density in the yoke and gap of the prototype MEMS quadrupole. Simulation results shown in the longitudinal plane in Fig. 4 demonstrate that fringing fields extend significantly beyond the region between the pole tips. This is a fundamental difference for our 3-D geometry and the main reason that 2-D approximations which assume a constant field profile along the longitudinal direction do not accurately model our case. Figure 5(a) shows the field intensity across the transverse axis of the electromagnet for transverse planes between the electromagnet center and $0.25 \mathrm{~mm}$ above the electromagnet center. Figure 5(b) shows the field gradient through the center of the electromagnet. It should be noted that while the integrated field gradient remains constant with respect to radial position throughout the electromagnet gap, the field gradient decreases and effective magnetic length increases with distance from the electromagnet pole tip. Figure 6 shows how the field gradient and magnetic length vary between the electromagnet center and edge of the magnetic gap. Simulation results converge with analytical calculations when yoke length is at least 4 times the magnet radius.

The prototype design left room for many improvements. When the windings are driven to produce a quadrupole field, the electromagnet saturates the poles near their connection to the yoke rather than at the tips due to magnetic flux fringing out of the pole surfaces. Driving current through the windings to produce a dipole field doubles the peak magnetic flux carried in the yoke, saturating the electromagnet well before the poles. A $15^{\circ}$ pole width taper $\left(7.5^{\circ}\right.$ on each side) was employed to retain the magnetic flux density towards the quadrupole pole tip, resulting in peak magnetic flux density in the yoke $0.26 \mathrm{~mm}$ from the pole tip for quadrupole fields. Tapering the pole to a wider base at the connection with the yoke brings the peak flux density closer to the pole tip and increases the peak field gradient, but also requires a wider yoke than can be accommodated in the $3 \mathrm{~mm} \times$ $3 \mathrm{~mm}$ prototype MEMS die footprint. This can be seen in Fig. 3(b), where the electromagnet yoke is as magnetized as the pole tips when the pole width taper is limited to $15^{\circ}$.

The relative thinness of the prototype quadrupole electromagnet yoke $(0.055 \mathrm{~mm})$ compared to the $0.3 \mathrm{~mm}$ magnet radius also results in large magnetic reluctance across the electromagnet gap compared to the

TABLE I. Simulated MEMS quadrupole field parameters in the center of the electromagnet driven to saturation. The prototype quadrupole electromagnet is typeset bold. Pole taper is optimized for each geometry to maximize peak field gradient.

\begin{tabular}{cccccc}
\hline \hline Magnet radius & Yoke length & Pole taper & Peak field gradient & Magnetic length & Winding current \\
\hline $\mathbf{0 . 3 0 0} \mathbf{~ m m}$ & $\mathbf{0 . 0 5 5} \mathbf{~ m m}$ & $\mathbf{7 . 5}^{\circ}$ & $\mathbf{2 5 3} \mathbf{~ T / m}$ & $\mathbf{0 . 4 7 7} \mathbf{~ m m}$ & $\mathbf{4 . 6} \mathbf{A}$ \\
$0.300 \mathrm{~mm}$ & $0.200 \mathrm{~mm}$ & $35^{\circ}$ & $1.0 \mathrm{kT} / \mathrm{m}$ & $0.504 \mathrm{~mm}$ & $9.6 \mathrm{~A}$ \\
$0.200 \mathrm{~mm}$ & $0.200 \mathrm{~mm}$ & $40^{\circ}$ & $2.2 \mathrm{kT} / \mathrm{m}$ & $0.387 \mathrm{~mm}$ & $6.1 \mathrm{~A}$ \\
$0.100 \mathrm{~mm}$ & $0.200 \mathrm{~mm}$ & $30^{\circ}$ & $6.1 \mathrm{kT} / \mathrm{m}$ & $0.281 \mathrm{~mm}$ & $2.9 \mathrm{~A}$ \\
$0.050 \mathrm{~mm}$ & $0.200 \mathrm{~mm}$ & $30^{\circ}$ & $10.0 \mathrm{kT} / \mathrm{m}$ & $0.245 \mathrm{~mm}$ & \\
\hline \hline
\end{tabular}




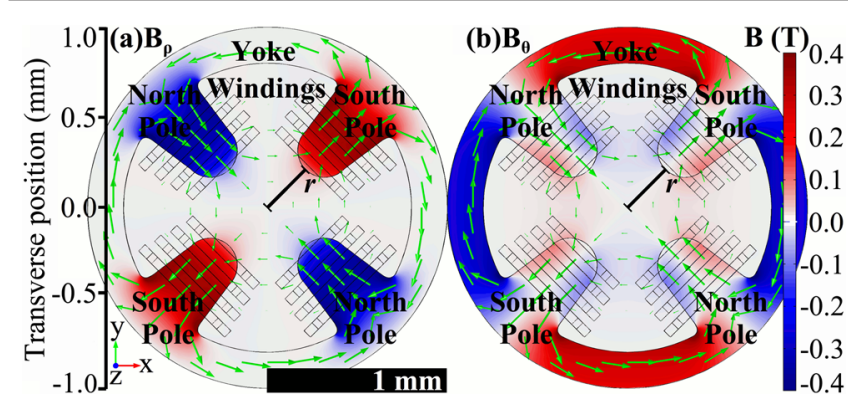

FIG. 3. Image of the 3-D FEM simulation of the prototype MEMS quadrupole in the 2-D plane through the center of the electromagnet yoke. Lines show the yoke and winding edges. Color shows the radial (a) and azimuth angle (b) vector component of the quadrupole magnetic field at $z=0 \mathrm{~mm}$ (quadrupole center) for $I=1 \mathrm{~A}$. Green arrows show the magnetic field direction (length shows log-scale intensity). The field gradient integrated down the longitudinal axis was $0.025 \mathrm{~T}$ at $1 \mathrm{~A}$.

3-D fringing reluctance behind the pole tip. The comparably large reluctance across the gap leads to significant 3-D fringing of the magnetic field before the pole tip and across the magnetic gap, reducing the peak field gradient in the center of the electromagnet while increasing the inductance and magnetic length. The effect of 3-D fringing spreading the field over the volume of the magnetic gap is shown qualitatively in Fig. 4 and quantitatively in Fig. 6, where the gradient in magnetic flux density is larger and magnetic length is shorter near the pole tip.

Recent fabrication process development has extended the maximum film thickness to $0.2 \mathrm{~mm}$ and the MEMS die footprint restrictions have been relaxed to $4 \mathrm{~mm} \times 4 \mathrm{~mm}$. Together with geometry optimizations, such as increasing the pole taper angle from $7.5^{\circ}$ to $35^{\circ}$ and increasing the yoke width from $0.2 \mathrm{~mm}$ to $0.5 \mathrm{~mm}$, a 4-fold improvement is projected in peak field gradient. We anticipate achieving

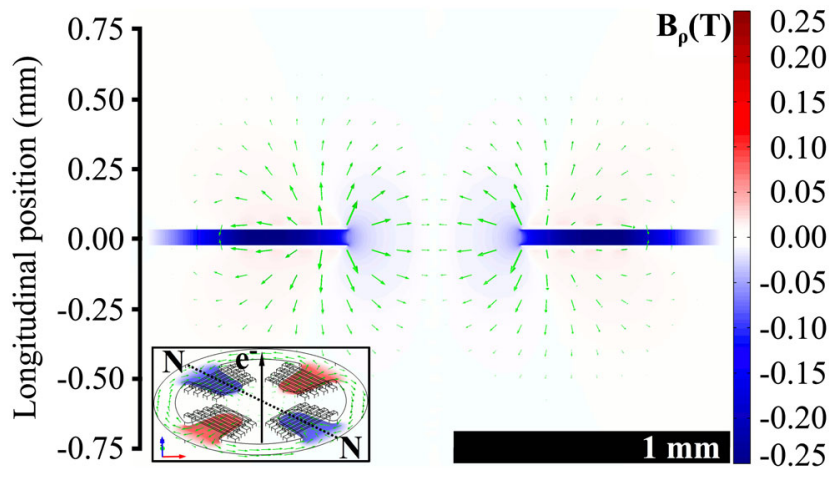

FIG. 4. Image of the 3-D FEM simulation of the prototype MEMS quadrupole in the 2-D plane through the two north pole tips and orthogonal to the electron beam axis (inset illustration). Color shows the radial vector component of the magnetic field for $I=1$ A. Green arrows show the 3-D direction of the magnetic field (arrow length shows log-scale intensity). It should be noted that magnetic fringing distributes much of the field over the volume beyond the two pole tips.
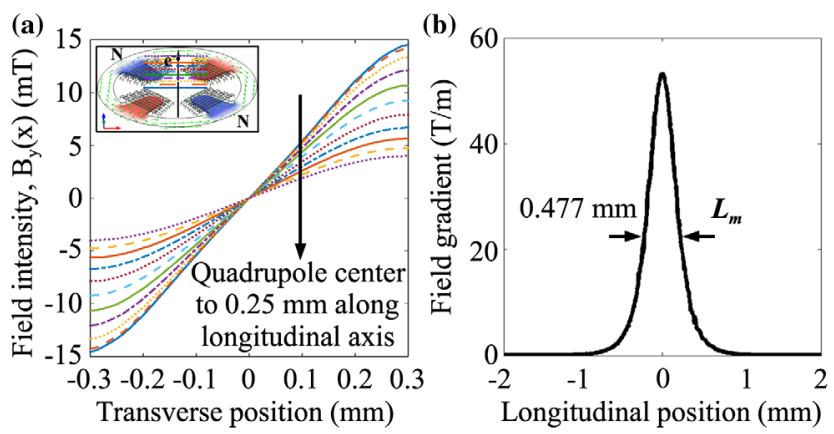

FIG. 5. FEM simulation of the prototype MEMS quadrupole. (a) Transverse quadrupole field intensity plotted across the $\mathrm{X}$-axis ( $B_{y}(x)$ between the pole tips) at longitudinal positions from the quadrupole center to $0.25 \mathrm{~mm}$ above the center. Note that the $\mathrm{X}$-axis does not intersect the poles. (b) Field gradient $\left(d B_{y} / d x\right)$ plotted down the electromagnet transverse center along the longitudinal (z) axis for $I=1$ A.

the performance figures projected in Table $\mathrm{I}$ in a future fabrication run.

\section{MULTI-POLE FIELD ANALYSIS}

Applications of particle beams require beam optics with a consistent field over the usable aperture and small undesired higher-order field content. Fringing fields resulting from the short yoke length lead to varying multi-pole coefficients as the electron traverses the focusing optic. Asymmetries in the manufactured quadrupole results in sextupole and octopole field components. The pole tip curvature, an imperfect hyperbolic shape truncated to

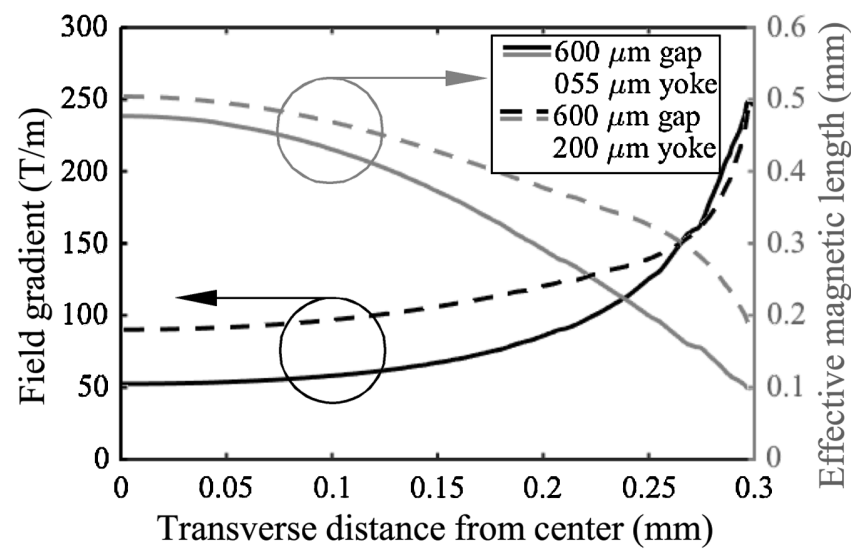

FIG. 6. Field gradient (black, $d B_{\theta}(r) / d r$ ) and effective magnetic length (gray) as a function of distance from the quadrupole center, calculated using FEM simulations of the quadrupole magnetic field at $1 \mathrm{~A}$. The pole tip is $0.3 \mathrm{~mm}$ from the center. The solid line shows the prototype 0.3 -mm-radius $0.055-\mathrm{mm}$ yoke length quadrupole and the dashed line shows an optimized 0.3-mm-radius 0.2-mm-yoke length quadrupole. Integrated field gradient remains constant across the magnetic gap. Variation in field gradient and magnetic length become more severe when the yoke length is much shorter than the magnetic gap. 


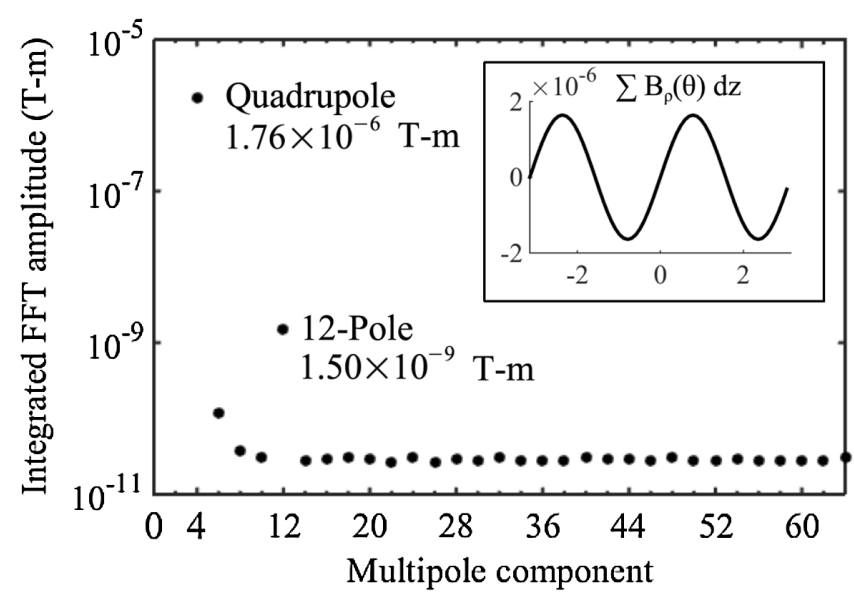

FIG. 7. Multi-pole moments of the prototype quadrupole field (inset) evaluated at the edge of the $0.1 \%$ good field region ( $0.072 \mathrm{~mm}$ from the center) and integrated through the longitudinal axis of the electromagnet. 4-pole is the quadrupole moment. The 12th, 20th, 28th, and higher order odd harmonics of the quadrupole moment result from truncating the infinite hyperbolic pole tips in 3-D. Other multi-pole components are a result of the finite mesh density and imperfect mesh symmetry of the FEM simulation.

accommodate the electromagnet windings, results in 12-pole and higher multi-pole field components.

The field vector and position data from the 3-D FEM simulation was converted to polar coordinates, and multipole analysis was performed using the radial field component for 128 radial distances from the center to the pole tip edge on 161 transverse planes from $1 \mathrm{~mm}$ below to $1 \mathrm{~mm}$ above the quadrupole center. Figure 7 shows the multi-pole analysis results for the prototype quadrupole, integrated through $2 \mathrm{~mm}$ of field data in the longitudinal direction. The dominant multi-pole component integrated down the longitudinal axis has amplitude of $1.76 \times 10^{-6} \mathrm{~T}-\mathrm{m}$ $0.072 \mathrm{~mm}$ from the electromagnet center, corresponding to a quadrupole field with $54 \mathrm{~T} / \mathrm{m}$ field gradient and $0.454 \mathrm{~mm}$ effective magnetic length.

We calculate the good field region by integrating the 12-pole to 64-pole multi-pole coefficients from FEM simulation down the longitudinal axis for each radial distance from the center and compare them to a percentage of the on-axis quadrupole field integrated down the longitudinal axis. Table II lists the radius of the region

TABLE II. Good field region radius from MEMS quadrupole simulation data. The prototype quadrupole is typeset bold.

\begin{tabular}{cccc}
\hline \hline $\begin{array}{c}\text { Magnet gap } \\
\text { radius }\end{array}$ & $\begin{array}{c}\text { Yoke } \\
\text { length }\end{array}$ & $\begin{array}{c}\text { 1\% Good field } \\
\text { radius }\end{array}$ & $\begin{array}{c}0.1 \% \text { Good field } \\
\text { radius }\end{array}$ \\
\hline $\mathbf{0 . 3 0 0} \mathbf{~ m m}$ & $\mathbf{0 . 0 5 5} \mathbf{~ m m}$ & $\mathbf{0 . 1 3 0} \mathbf{~ m m}$ & $\mathbf{0 . 0 7 2} \mathbf{~ m m}$ \\
$0.300 \mathrm{~mm}$ & $0.200 \mathrm{~mm}$ & $0.197 \mathrm{~mm}$ & $0.110 \mathrm{~mm}$ \\
$0.200 \mathrm{~mm}$ & $0.200 \mathrm{~mm}$ & $0.124 \mathrm{~mm}$ & $0.069 \mathrm{~mm}$ \\
$0.100 \mathrm{~mm}$ & $0.200 \mathrm{~mm}$ & $0.059 \mathrm{~mm}$ & $0.035 \mathrm{~mm}$ \\
$0.050 \mathrm{~mm}$ & $0.200 \mathrm{~mm}$ & $0.021 \mathrm{~mm}$ & $0.011 \mathrm{~mm}$ \\
\hline \hline
\end{tabular}

TABLE III. Multi-pole components from FEM simulation data normalized to the quadrupole field moment for MEMS quadrupoles. Multi-pole component data is evaluated at the edge of the $0.1 \%$ good field region for each simulated quadrupole. The prototype quadrupole is typeset bold. The 20-pole field component was below the resolution of the simulation for the prototype quadrupole.

\begin{tabular}{cccc}
\hline \hline Magnet gap radius & Yoke length & 12-pole & 20-pole \\
\hline $\mathbf{0 . 3 0 0} \mathbf{~ m m}$ & $\mathbf{0 . 0 5 5} \mathbf{~ m m}$ & $\mathbf{9} \times \mathbf{1 0}^{-\mathbf{4}}$ & $<\mathbf{2} \times \mathbf{1 0}^{-\mathbf{5}}$ \\
$0.300 \mathrm{~mm}$ & $0.200 \mathrm{~mm}$ & $8 \times 10^{-4}$ & $3 \times 10^{-4}$ \\
$0.200 \mathrm{~mm}$ & $0.200 \mathrm{~mm}$ & $8 \times 10^{-4}$ & $3 \times 10^{-5}$ \\
$0.100 \mathrm{~mm}$ & $0.200 \mathrm{~mm}$ & $7 \times 10^{-4}$ & $4 \times 10^{-5}$ \\
$0.050 \mathrm{~mm}$ & $0.200 \mathrm{~mm}$ & $8 \times 10^{-4}$ & $4 \times 10^{-4}$ \\
\hline \hline
\end{tabular}

(Good field radius) where sums of the multi-pole field components are less than $1 \%$ and $0.1 \%$ the magnitude of the quadrupole field moment.

Table III details the multi-pole analysis results evaluated at the edge of the $0.1 \%$ good field region for the prototype quadrupole electromagnet (typeset bold) and four quadrupole electromagnets optimized for peak gradient. 12-pole and 20-pole list the intensity of higher-order multi-pole field components relative to the quadrupole field moment. Higher-order multi-pole field content was correlated to the shape of the yoke pole tips, increasing as the hyperbolicshaped tip width was truncated in smaller-gap electromagnets to provide space for the electromagnet windings. Multi-pole components higher than 12-pole were below the simulation resolution for the prototype quadrupole but resolvable for the optimized quadrupoles.

\section{POWER EFFICIENCY AND DUTY CYCLE}

The low impedance $(58 \mathrm{~m} \Omega, 30 \mathrm{nH})$ of these MEMS electromagnets facilitates circuit-limited driving frequencies up to $2 \mathrm{MHz}$, allowing pulse-to-pulse reconfiguration of the magnetic field or short-duty-cycle operation. Driving the electromagnet windings with short-duty cycle current pulses dramatically reduces the average power dissipated by the electromagnet and relaxes the thermal constraints on the electromagnets. Figure 8 shows the expected average power dissipation of the MEMS quadrupole electromagnet driven by $1 \mathrm{~A}$ pulses at $960 \mathrm{~Hz}$ for pulse widths between $1 \mu \mathrm{s}$ and $1 \mathrm{~ms}$ ( $0.1 \%$ to $96 \%$ duty cycle), calculated using the measured impedance of the $0.3 \mathrm{~mm}$ radius MEMS electromagnet up to $1 \mathrm{MHz}$. Despite the increased dissipation due to skin effect and eddy current losses, reducing the duty cycle to $0.1 \%$ reduces power dissipation by a factor of $>750$, allowing $>25$ times the drive current compared to continuous operation.

\section{ELECTRON BEAM EXPERIMENTS}

To demonstrate beam focusing and steering, the MEMS quadrupole was mounted in the path of an electron beam 


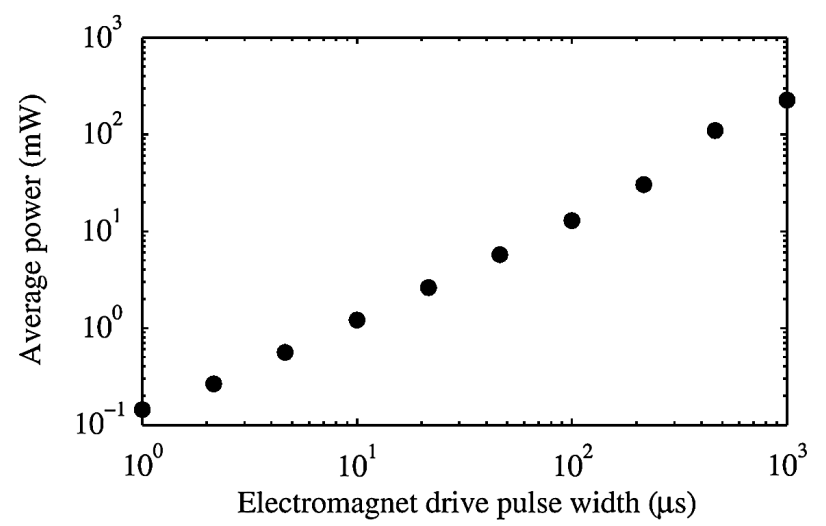

FIG. 8. Average power dissipation calculated for a short duty cycle $(0.1 \%-96 \%) 1 \mathrm{~A}$ pulse train at $960 \mathrm{~Hz}$ using complex impedance measurements from the prototype quadrupole.

and powered in dipole or quadrupole configurations (Fig. 9). The electron beam used in this experiment is generated using a short UV laser pulse illuminating a photocathode embedded in a static electric field (DC photogun). A solenoid electromagnet adjusts the beam focus exiting the photocathode, and a set of steering electromagnets adjust position and angle during an $835 \mathrm{~mm}$ drift length to an experiment chamber. The chamber houses the MEMS quadrupole behind a pair of micrometer-mounted orthogonal 0.02-mm slits (Thorlabs S20R) that have been stripped of anodizing and iron oxide coatings to form an electron beam aperture. After another $115 \mathrm{~mm}$ drift length, an imaging system composed of a Chevron micro-channel plate (MCP) intensifier, phosphor

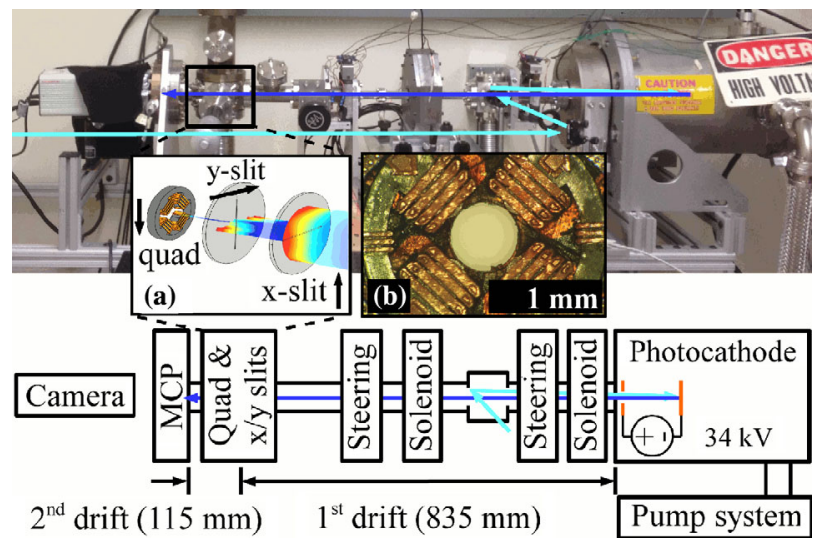

FIG. 9. Photograph and illustration of the electron beam experiment. Inset (a) shows an illustration of the inside of the experiment chamber with an electron beam (colored) entering the chamber from the right, striking a horizontal slit (x-slit) that is inserted into the chamber from below, a vertical slit (y-slit) that is inserted into the chamber from the left, and passing through a MEMS quadrupole (quad) that is inserted into the chamber from above. Inset (b) shows a photograph of a MEMS quadrupole. Cyan arrows illustrate the UV laser path from left to right and blue arrows illustrate the electron beam path from right to left. screen, and cooled CCD camera (Hamamatsu Flash 2.8) records the beam position and shape.

We acquired a background shot with the electron beam turned off for each image, which was subtracted from the data before calculating beam centroid and root mean square (RMS) size. The experiment used a slightly under-focused $34 \mathrm{keV}$ sub-pC electron beam pulsed at $960 \mathrm{~Hz}$ repetition rate. The aperture central position was obtained by switching the MEMS electromagnet on and off in quadrupole configuration and adjusting the position until the beam location did not change. Each measurement consisted of 25 images taken with $250 \mathrm{~ms}$ exposure time.

\section{A. Electron beam steering}

To measure beam steering performance, the electromagnet current was stepped from $-1.5 \mathrm{~A}$ to $+1.5 \mathrm{~A}$ and back to $-1.5 \mathrm{~A}$ in $0.5 \mathrm{~A}$ increments in an $\mathrm{x}$-dipole field configuration (two adjacent poles energized as North and two adjacent poles energized as South) and then repeated in a y-dipole configuration. Figure 10 shows measured data for the beam centroid steered right, left, down, and up. Steering the $34 \mathrm{keV}$ electron beam with the electromagnet configured in dipole mode at $1 \mathrm{~A}$ resulted in a $1.2 \mathrm{~mm}$ deflection after a $115 \mathrm{~mm}$ drift distance, corresponding to a $10.8 \mathrm{mT}$ dipole field using the small angle approximation. Electromagnet hysteresis when changing steering directions and third order nonlinear coefficients measurements were at the limits of the experiment resolution $(0.042 \mathrm{~mm})$.

\section{B. Quadrupole field mapping}

Spatially mapping a rapidly changing magnetic field in a sub-mm electromagnet gap presents significant challenges. Traditional methods such as a measuring vibrations induced by current pulses in a thin beryllium copper wire

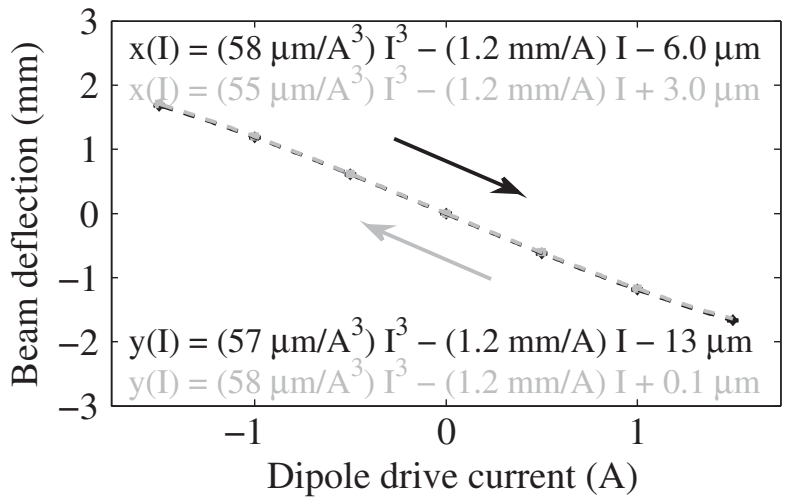

FIG. 10. Measured beam steering right (top black), left (top gray), down (bottom black) and up (bottom gray) across the MCP. Black steering data was taken while increasing the current and grey data was taken while reducing the current. Hysteresis and nonlinearity is less than the measurement error, resulting in the $y(I)$ line overlaying most of the other lines. 


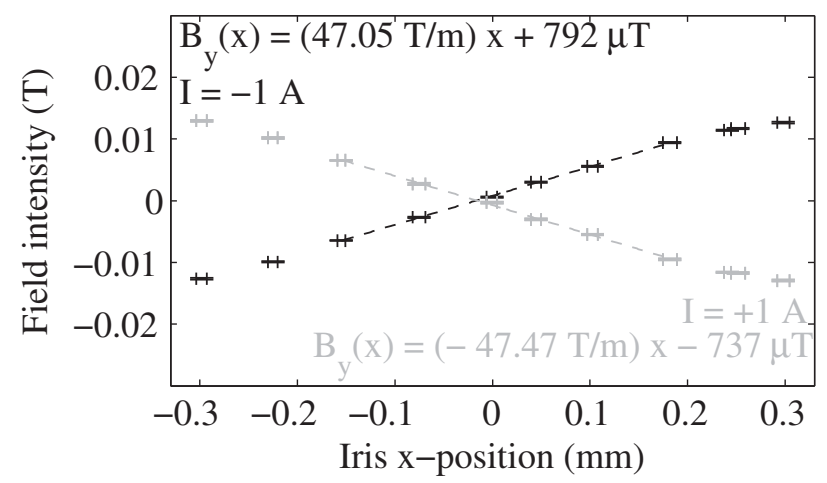

FIG. 11. X-axis transverse field profile measured with a $0.02 \mathrm{~mm}$ electron beam probe. Error bars and regression lines show the field across the center of the gap. $54.3 \mathrm{~T} / \mathrm{m}$ gradients at $1 \mathrm{~A}$ are expected from FEM simulations.

appear to be complicated at these scales by acoustic dispersion and traditional rotating coils and hall-effect probes are not small enough to measure inside the gap with $0.010 \mathrm{~mm}$-scale spatial resolution and $\mathrm{mT}$-scale field intensity resolution. More exotic techniques such as nuclear magnetic resonance coils or microfabricated Hall-effect probes proved too challenging to achieve successful measurements with. The deflection of a probe electron beam, however, directly measures the integrated field through a transverse position of the electromagnet gap.

Field uniformity measurements were taken in quadrupole configuration (adjacent poles energized as opposite polarities) from $-1 \mathrm{~A}$ to $1 \mathrm{~A}$ current in $0.5 \mathrm{~A}$ increments using an electron beam probe. For each electromagnet current dataset, the 2-slit aperture was stepped in equal intervals across the entire electromagnet bore. The magnetic field value was obtained by measuring the electron beam deflection for each aperture position and using the effective magnetic length from FEM simulations. Figure 11 (Fig. 12) shows the field gradient across the horizontal (vertical) transverse direction calculated from the measurements. $47 \mathrm{~T} / \mathrm{m}$ and $57 \mathrm{~T} / \mathrm{m}$ were measured across the $\mathrm{x}$ and $\mathrm{y}$-axis, respectively.

The field gradient, shown in Fig. 13, scales linearly with current, as expected. Measured hysteresis is less than the measurement variance. The difference between the horizontal and vertical field gradient could be due to poor control of the quadrupole electromagnet axis in the experimental chamber. The field gradient calculated from the measured beam displacement assumes that the beam travels normal to the transverse field of the quadrupole through an effective magnetic length of $0.477 \mathrm{~mm}$. The experiment obstructed observation of electromagnet orientation inside the chamber and the azimuth angle was hand-set to an angle that passed the beam. The edges of the $0.60 \mathrm{~mm}$ hole in the $0.55 \mathrm{~mm}$ thick substrate would begin to obstruct the centered $0.02 \mathrm{~mm}$ beam at an azimuth misalignment of $47^{\circ}$. Simulations indicate that a $20^{\circ}$ azimuth error in the

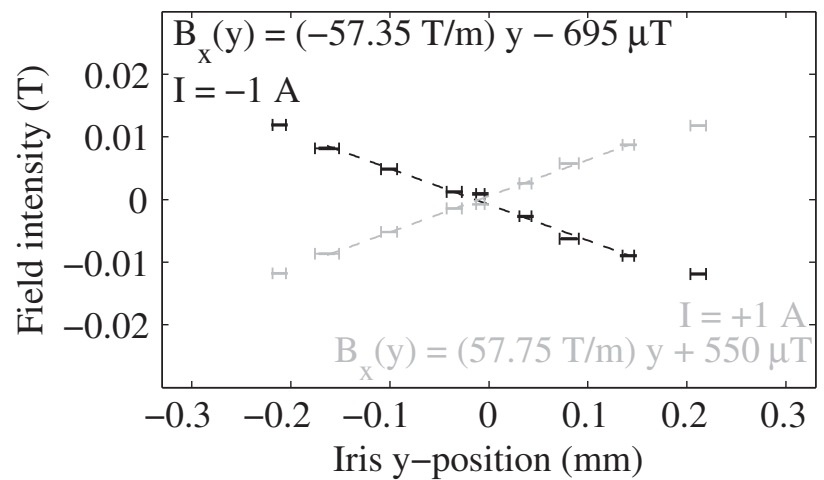

FIG. 12. Y-axis transverse field profile measured with a $0.02 \mathrm{~mm}$ electron beam probe. Error bars and regression lines show the field across the center of the gap. $54.3 \mathrm{~T} / \mathrm{m}$ gradients at $1 \mathrm{~A}$ are expected from FEM simulations.

experiment would reduce the integrated field gradient on the beam path for the y-oriented field vector, reducing the apparent $\mathrm{x}$-axis transverse field gradient shown in Fig. 11.

Other potential causes of the mismatch between the measured quadrupole field on each axis include manufacturing defects in the electromagnet geometry or nonuniform properties in the magnetic yoke. The geometric accuracy of the yoke measured using scanning electron micrographs of the device was within $0.001 \mathrm{~mm}$ and chemical analysis of the magnetic alloy composition with energy dispersive $\mathrm{x}$-ray spectroscopy on the surface of the yoke during manufacturing was within $1 \%$ of $\mathrm{Ni}_{80} \mathrm{Fe}_{20}$. The microscale magnetic properties of the yoke and the magnetic alloy composition within the yoke volume were not measured, however.

\section{Electron beam focusing}

The measured field gradient and RMS beam width at different electromagnet currents can be used to calculate beam parameters in a quadrupole scan measurement, demonstrating that the MEMS quadrupoles work as

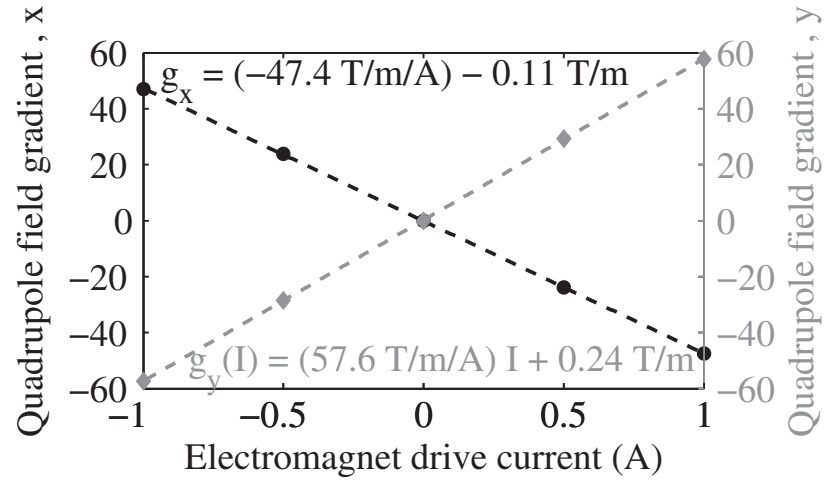

FIG. 13. Transverse field gradient measured with a $0.02 \mathrm{~mm}$ electron beam probe. Gradient measured along the horizontal axis is black and measured along the vertical axis is gray. 


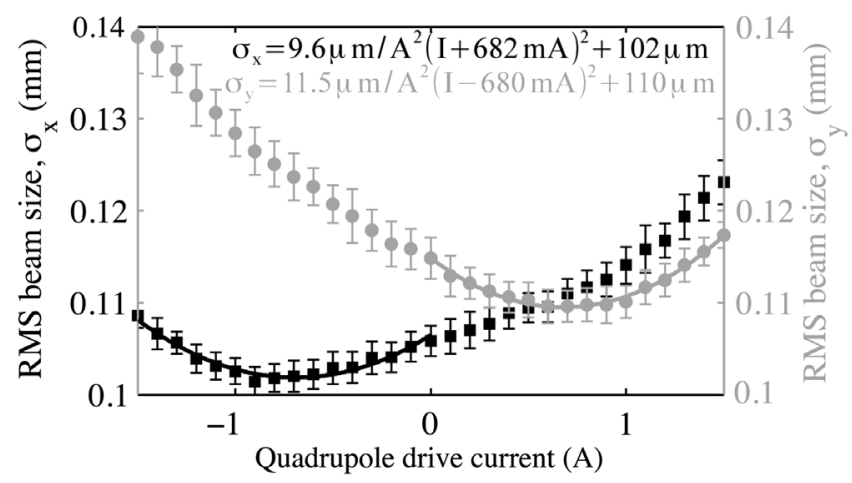

FIG. 14. Beam width after focusing with the quadrupole electromagnet powered in quadrupole configuration. Horizontal RMS width is black and vertical RMS width is gray.

conventional magnetic optics. To measure the performance of the quadrupole focusing the beam, the quadrupole was repositioned and reoriented, and the electromagnet current was configured for quadrupole mode and stepped from $-1.5 \mathrm{~A}$ to $+1.5 \mathrm{~A}$ and back to $-1.5 \mathrm{~A}$ in $0.1 \mathrm{~A}$ increments. The measured RMS beam width is shown in Fig. 14, and a focused beam waist is obtained at the MCP plane in the $\mathrm{y}$-axis for $I=0.9 \mathrm{~A}$ and in the $\mathrm{x}$-axis for $I=-0.9 \mathrm{~A}$. The beam expands slightly between the first (horizontal) and second (vertical) slit, resulting in the small observed asymmetry.

The data can be fitted using the gradient extracted from the quadrupole field mapping measurements $(57.6 \mathrm{~T} / \mathrm{m})$ to obtain an estimate for the beam phase space parameters at the quadrupole entrance. Using the transfer matrix of a thin quadrupole of focal distance $f$ and drift length $l_{d}$ between the quadrupole and MCP screen, and representing the electron beam with its transverse phase space parameters ( $x$ for position, $x^{\prime}$ for momentum or angular deviation in the small angle approximation, and $\epsilon=\sqrt{\langle x x\rangle\left\langle x^{\prime} x^{\prime}\right\rangle-\left\langle x x^{\prime}\right\rangle^{2}}$ for RMS emittance) in the small angle approximation, we can write for the RMS beam size at the imaging screen $\sigma_{x, s}^{2}=\operatorname{var}\left(x_{s}\right)$ in the transverse horizontal axis:

$$
\sigma_{x, s}^{2}=\left(1-\frac{l_{d}}{f}\right)^{2} \sigma_{x}^{2}+l_{d}^{2} \sigma_{x^{\prime}}^{2}+2 l_{d}\left(1-\frac{l_{d}}{f}\right) \sigma_{x x^{\prime}} .
$$

The beam parameters at the quadrupole from the fit of the data yield RMS beam width $\sigma_{x}=0.017 \mathrm{~mm}$, $\sigma_{y}=0.021 \mathrm{~mm}$ and angular divergence $\sigma_{x^{\prime}}=0.9 \mathrm{mrad}$, $\sigma_{y^{\prime}}=1.0 \mathrm{mrad}$, which is in good agreement with the beam parameters expected following the $20 \mu \mathrm{m}$ aperture and validates our model for the MEMS quadrupole performance.

\section{CONCLUSIONS}

MEMS quadrupole electromagnets with sub-mm magnetic gap were designed, fabricated, and tested with a $34 \mathrm{keV}$ electron beam. The field gradient increases and the inductance decreases considerably as the size of these beam optics are reduced into the micro-scale. Simple scaling of the prototype results presented in this paper indicates that multi-kT/m gradients can be obtained using MEMS fabrication techniques. Such MEMS quadrupoles hold the potential to revolutionize the field of charged particle optics with a wide range of applications including electron microscopes and compact light sources.

\section{ACKNOWLEDGMENTS}

The authors would like to thank the UCLA Nanoelectronics Research Facility (Nanolab) and Integrated System Nanofabrication Cleanroom (ISNC) for use of their extensive fabrication facilities, Radiabeam Technologies for the use of their electron gun, and the referees of this paper for their valuable time. We gratefully acknowledge the support by the Defense Advanced Research Projects Agency under Grant No. N66001-12-1-4209, the National Science Foundation under Grants No. 1350034 and No. PHY 1415583, and the W. M. Keck Foundation.

[1] O. Krivanek, P. Nellist, N. Dellby, M. Murfitt, and Z. Szilagyi, Towards sub-0.5 A electron beams, Ultramicroscopy 96, 229 (2003).

[2] R. K. Li, W. Huang, Y. Du, L. Yan, Q. Du, J. Shi, J. Hua, H. Chen, T. Du, H. Xu, and C. Tang, Single-shot continuously time-resolved $\mathrm{MeV}$ ultrafast electron diffraction, Rev. Sci. Instrum. 81, 036110 (2010).

[3] E. Pedroni, R. Bacher, H. Blattmann, T. Böhringer, A. Coray, A. Lomax, S. Lin, G. Munkel, S. Scheib, U. Schneider et al., The $200 \mathrm{MeV}$ proton therapy project at the Paul Scherrer Institute: Conceptual design and practical realization, Med. Phys. 22, 37 (1995).

[4] T. Ishikawa, H. Aoyagi, T. Asaka, Y. Asano, N. Azumi, T. Bizen, H. Ego, K. Fukami et al., A compact X-ray freeelectron laser emitting in the sub-ångström region, Nat. Photonics 6, 540 (2012).

[5] M. Xie, Exact and variational solutions of 3D eigenmodes in high gain FELs, Nucl. Instrum. Methods Phys. Res., Sect. A 445, 59 (2000).

[6] R. Schoenlein, W. Leemans, A. Chin, P. Volfbeyn, T. Glover, P. Balling, M. Zolotrev, K.-J. Kim, S. Chattopadhyay, and C. Shank, Femtosecond X-ray Pulses at $0.4 \AA$ Generated by $90^{\circ}$ Thomson Scattering: A Tool for Probing the Structural Dynamics of Materials, Science 274, 236 (1996).

[7] I. V. Pogorelsky, I. Ben-Zvi, T. Hirose, S. Kashiwagi, V. Yakimenko, K. Kusche, P. Siddons, J. Skaritka, T. Kumita, A. Tsunemi, T. Omori, J. Urakawa, M. Washio, K. Yokoya, T. Okugi, Y. Liu, P. He, and D. Cline, Demonstration of $8 \times 10^{18}$ photons/second peaked at $1.8 \AA$ in a relativistic Thomson scattering experiment, Phys. Rev. ST Accel. Beams 3, 090702 (2000).

[8] J. K. Lim, P. Frigola, G. Travish, J. B. Rosenzweig, S. G. Anderson, W. J. Brown, J. S. Jacob, C. L. Robbins, and 
A. M. Tremaine, Adjustable, short focal length permanentmagnet quadrupole based electron beam final focus system, Phys. Rev. ST Accel. Beams 8, 072401 (2005).

[9] M. Haider, S. Uhlemann, E. Schwan, H. Rose, B. Kabius, and K. Urban, Electron microscopy image enhanced, Nature (London) 392, 768 (1998).

[10] R. K. Li and P. Musumeci, Single-Shot MeV Transmission Electron Microscopy with Picosecond Temporal Resolution, Phys. Rev. Applied 2, 024003 (2014).

[11] N. Barov and J.B. Rosenzweig, Propagation of short electron pulses in underdense plasmas, Phys. Rev. E 49, 4407 (1994).

[12] N. Barov, M. E. Conde, W. Gai, and J. B. Rosenzweig, Propagation of Short Electron Pulses in a Plasma Channel, Phys. Rev. Lett. 80, 81 (1998).

[13] R. Weingartner, M. Fuchs, A. Popp, S. Raith, S. Becker, S. Chou, M. Heigoldt, K. Khrennikov, J. Wenz, T. Seggebrock, B. Zeitler, Z. Major, J. Osterhoff, F. Krausz, S. Karsch, and F. Grüner, Imaging laser-wakefieldaccelerated electrons using miniature magnetic quadrupole lenses, Phys. Rev. ST Accel. Beams 14, 052801 (2011).

[14] B. Naranjo, A. Valloni, S. Putterman, and J. B. Rosenzweig, Stable Charged-Particle Acceleration and Focusing in a Laser Accelerator Using Spatial Harmonics, Phys. Rev. Lett. 109, 164803 (2012).

[15] J. Breuer and P. Hommelhoff, Laser-Based Acceleration of Nonrelativistic Electrons at a Dielectric Structure, Phys. Rev. Lett. 111, 134803 (2013).

[16] T. Plettner and R. L. Byer, Proposed dielectric-based microstructure laser-driven undulator, Phys. Rev. ST Accel. Beams 11, 030704 (2008).

[17] J. Harrison, A. Joshi, J. Lake, R. Candler, and P. Musumeci, Surface-micromachined magnetic undulator with period length between $10 \mu \mathrm{m}$ and $1 \mathrm{~mm}$ for advanced light sources, Phys. Rev. ST Accel. Beams 15, 070703 (2012).

[18] B. Peterson, O. Oniku, W. Patterson, D. Le Roy, A. Garraud, F. Herrault, N. Dempsey, D. Arnold, and M. Allen, Technology development for short-period magnetic undulators, Phys. Procedia 52, 36 (2014).

[19] P. Musumeci, J. T. Moody, R. J. England, J. B. Rosenzweig, and T. Tran, Experimental Generation and Characterization of Uniformly Filled Ellipsoidal Electron-Beam Distributions, Phys. Rev. Lett. 100, 244801 (2008).

[20] Y. Ding, A. Brachmann, F.-J. Decker, D. Dowell, P. Emma, J. Frisch, S. Gilevich, G. Hays, P. Hering, Z. Huang,
R. Iverson, H. Loos, A. Miahnahri, H.-D. Nuhn, D. Ratner, J. Turner, J. Welch, W. White, and J. Wu, Measurements and Simulations of Ultralow Emittance and Ultrashort Electron Beams in the Linac Coherent Light Source, Phys. Rev. Lett. 102, 254801 (2009).

[21] T. Rao and D. H. Dowell, An Engineering Guide To Photoinjectors, [arXiv:1403.7539].

[22] J. Harrison, O. Paydar, Y. Hwang, J. Wu, E. Threlkeld, P. Musumeci, and R. Candler, Fabrication process for thickfilm micromachined multi-pole electromagnets, J. Microelectromech. Syst. 23, 505 (2014).

[23] T. Eichner, F. Grüner, S. Becker, M. Fuchs, D. Habs, R. Weingartner, U. Schramm, H. Backe, P. Kunz, and W. Lauth, Miniature magnetic devices for laser-based, tabletop free-electron lasers, Phys. Rev. ST Accel. Beams 10, 082401 (2007).

[24] M. Winkler, V. Chichkine, K.-H. Behr, H. Geissel, S. Eliseev, A. Kalimov, G. Li, G. Münzenberg, W. Plaß, C. Scheidenberger, Z. Wang, H. Weick, and H. Wollnik, Development and test of iron-free quadrupole lenses with high magnetic flux densities, Nucl. Instrum. Methods Phys. Res, Sec. B 204, 454 (2003).

[25] V. Rebrov, A. Ponomarev, V. Palchik, and N. Melnik, The new design of magnetic quadrupole lens doublet manufactured from a single piece, Nucl. Instrum. Methods, Phys. Res. B 260, 34 (2007).

[26] Y. Shi, S. Ardanuç, and A. Lal, in Micro-Einzel lens for wafer-integrated electron beam actuation, MEMS (IEEE, New York, 2013), p. 189.

[27] R. Candler, W.-T. Park, H. Li, G. Yama, A. Partridge, M. Lutz, and T. Kenny, Single wafer encapsulation of MEMS devices, IEEE Trans. Adv. Packag. 26, 227 (2003).

[28] P. Van Kessel, L. Hornbeck, R. Meier, and M. Douglass, A MEMS-based projection display, Proc. IEEE 86, 1687 (1998).

[29] S. Knappe, V. Shah, P. Schwindt, L. Hollberg, J. Kitching, L.-A. Liew, and J. Moreland, A microfabricated atomic clock, Appl. Phys. Lett. 85, 1460 (2004).

[30] M. Glickman, J. Harrison, T. Niblock, P. Tseng, and J. Judy, in Proceedings of the Solid-State Sensors, Actuators and Microsystems Workshop, High permeability Permalloy for MEMS, (Transducer Research Foundation, San Diego, 2010), p. 328.

[31] M. Glickman, P. Tseng, J. Harrison, T. Niblock, I. B. Goldberg, and J. W. Judy, High-performance lateralactuating magnetic MEMS switch, J. Microelectromech. Syst. 20, 842 (2011). 